\title{
In-situ Sensing Using Mass Spectrometry and its Use for Run-To-Run Control on a W-CVD Cluster Tool
}

\author{
T. Gougousi ${ }^{1}$, R. Sreenivasan ${ }^{2}$, Y. Xu ${ }^{1}$, L. Henn-Lecordier ${ }^{1}$, G. W. Rubloff ${ }^{1}$, \\ J. N. Kidder, Jr. ${ }^{1}$, and E. Zafiriou ${ }^{2}$ \\ ${ }^{1}$ Institute for Systems Research and Department of Materials and Nuclear Engineering \\ ${ }^{2}$ Institute for Systems Research and Department of Chemical Engineering \\ University of Maryland, College Park, MD 20742-3285
}

\begin{abstract}
A 300 amu closed-ion-source RGA (Leybold-Inficon Transpector 2) sampling gases directly from the reactor of an ULVAC ERA-1000 cluster tool has been used for real time process monitoring of a W CVD process. The process involves $\mathrm{H}_{2}$ reduction of $\mathrm{WF}_{6}$ at a total pressure of $67 \mathrm{~Pa}(0.5$ torr $)$ to produce $\mathrm{W}$ films on $\mathrm{Si}$ wafers heated at temperatures around $350^{\circ} \mathrm{C}$. The normalized RGA signals for the $\mathrm{H}_{2}$ reagent depletion and the $\mathrm{HF}$ product generation were correlated with the $\mathrm{W}$ film weight as measured post-process with an electronic microbalance for the establishment of thin-film weight (thickness) metrology. The metrology uncertainty (about $7 \%$ for the HF product) was limited primarily by the very low conversion efficiency of the W CVD process (around 2-3\%). The HF metrology was then used to drive a robust run-to-run control algorithm, with the deposition time selected as the manipulated (or controlled) variable. For that purpose, during a 10 wafer run, a systematic process drift was introduced as a $-5^{\circ} \mathrm{C}$ processing temperature change for each successive wafer, in an otherwise unchanged process recipe. Without adjustment of the deposition time the $\mathrm{W}$ film weight (thickness) would have declined by about $50 \%$ by the $10^{\text {th }}$ wafer. With the aid of the process control algorithm, an adjusted deposition time was computed so as to maintain constant HF sensing signal, resulting in weight (thickness) control comparable to the accuracy of the thickness metrology. These results suggest that in-situ chemical sensing, and particularly mass spectrometry, provide the basis for wafer state metrology as needed to achieve run-to-run control. Furthermore, since the control accuracy was consistent with the metrology accuracy, we anticipate significant improvements for processes as used in manufacturing, where conversion rates are much higher (40-50\%) and corresponding signals for metrology will be much larger.
\end{abstract}

Keyword: Run-to-Run control, W-CVD, Mass Spectrometry, Metrology, IMC, EWMA

\section{INTRODUCTION}

Advanced Process Control (APC) provides benefits in two areas: (1) fault management (error identification/classification) and (2) course correction (set point tracking). While these have been a mainstay in the chemical industry for some time, they are only recently being developed and implemented in semiconductor manufacturing processes. As the industry is moving to larger wafer sizes and shrinking device sizes, loss of processed wafers due to delayed detection of equipment/process problems can have severe financial implications. As a result, the need for the introduction of APC in this industry has been recognized [1] and some effort has been devoted to the transfer of technological know-how from other engineering disciplines. This transfer is, however, far from trivial mainly due to the absence of accurate models for semiconductor processes and lack of sensor-based metrology implementation [2].

In this article we present the use of mass spectrometry for real-time process monitoring of a W CVD process, the establishment of thin film thickness metrology and its application for Run-to-Run process control.

\section{EXPERIMENTAL}

An ULVAC ERA-1000 cluster tool was used for deposition of $\mathrm{W}$ films on $\mathrm{Si}$ wafers via $\mathrm{H}_{2}$ reduction of $\mathrm{WF}_{6}$. The reagent flow rates were $40 \mathrm{sccm}$ for $\mathrm{H}_{2}$ and

CP550, Characterization and Metrology for ULSI Technology: 2000 International Conference, edited by D. G. Seiler, A. C. Diebold, T. J. Shaffner, R. McDonald, W. M. Bullis, P. J. Smith, and E. M. Secula (C) 2001 American Institute of Physics 1-56396-967-X/01/\$18.00 
$10 \mathrm{sccm}$ for $\mathrm{WF}_{6}$, at a total pressure of $67 \mathrm{~Pa}(0.5$ torr $)$ and actual wafer temperatures around $350^{\circ} \mathrm{C}$. For specificity in this paper, we always refer to setpoint temperatures on the tool controller. However, we determined experimentally that actual wafer temperatures are significantly $\left(100-150^{\circ} \mathrm{C}\right)$ below the setpoint temperature entered on the tool recipe; this should be kept in mind in comparing these results to prior reports in the literature.

The process initially deposited a $\mathrm{W}$ seed layer on the $\mathrm{Si}$ surface through the nucleation reactions:

$$
\begin{gathered}
2 \mathrm{WF}_{6}+3 \mathrm{Si} \rightarrow 2 \mathrm{~W}(\mathrm{~s})+3 \mathrm{SiF}_{4} \uparrow \\
\mathrm{WF}_{6}+3 \mathrm{Si}+\mathrm{H}_{2} \rightarrow 2 \mathrm{~W}(\mathrm{~s})+2 \mathrm{SiHF}_{3} \uparrow
\end{gathered}
$$

Once the $\mathrm{W}$ seed layer was formed, it provided the activated surface sites $\left(\mathrm{W}^{*}(\mathrm{~s})\right)$ for the reaction through dissociative adsorption of $\mathrm{WF}_{6}$ and $\mathrm{F}$ atom removal as the HF volatile product:

$$
\begin{aligned}
\mathrm{W}^{*}(\mathrm{~s})+\mathrm{WF}_{6} \rightarrow \mathrm{WF}_{6}^{*}+\mathrm{W}(\mathrm{s}) \\
\mathrm{WF}_{6} *+3 \mathrm{H}_{2} \rightarrow \mathrm{W}(\mathrm{s})+6 \mathrm{HF} \uparrow
\end{aligned}
$$

The process operating pressure, developed by Ulvac for a selective $\mathrm{W}$ CVD process, was significantly lower than that for blanket W CVD as now routinely practiced in the industry, about $5 \mathrm{kPa}$ (or 40 torr). As a result the reagent conversion was limited to $2-3 \%$, making this process much more challenging for both metrology and control than should be expected for commercial blanket W CVD processes.

A Leybold-Inficon Transpector 2 CIS-RGA sampling gases directly from the reactor was used to mass analyze the composition of the process gas mixture. The RGA was used to monitor the partial pressures of both reagents $\left(\mathrm{H}_{2}\right.$ and $\left.\mathrm{WF}_{6}\right)$ and the $\mathrm{HF}$ product, in addition to a number of other species associated with impurity concerns.

\section{RGA-BASED METROLOGY}

The RGA signals for the HF product and the $\mathrm{H}_{2}$ reagent, corrected for background and normalized for sensor and/or process drifts were correlated with the $\mathrm{W}$ film weight as measured post-process with an electronic microbalance for the establishment of thin film weight (thickness) metrology. From the two, the HF based metrology exhibited better accuracy ( $7 \%)$ and was thus selected for the Run-to-Run control experiments. A detailed account of the metrology development and demonstration can be found elsewhere [3].

\section{THE CONTROL PROBLEM}

The main objective of a process control strategy is to keep the value of the control variable within a target range in the presence of process disturbances, which are manifested as sudden step changes (called shifts here), and/or continuous monotonic changes (defined here as drifts). In our experiment the parameter of interest (primary control variable) was the amount of W deposited on the wafer, expressed either as film weight or film thickness. A parameter directly related to it through in-situ sensor-based metrology was the normalized HF mass spectrometer signal, and will be called the secondary control variable. The process variables, such as the reagent flow rates, the process pressure, the wafer, and the deposition time had a direct effect on the $\mathrm{W}$ film weight deposited on the wafer and could thus be used as manipulated (or controlled) variables to keep the process on track. However, the reagent flow rates and the process pressure were quickly eliminated as potential manipulated variables due to their very limited effect on the deposition rate in the process window. Temperature variations had a quite significant effect on the deposition rate but it we decided to use it as an artificial disturbance instead so as to test the control system. As a result, the deposition time was left as the only potential manipulated variable, giving us a Single Input-Single Output (SISO) control scheme.

The objective of this work was to develop a control methodology, which exploited in-situ sensor-based metrology to maintain the $\mathrm{W}$ film weight at the target value in the presence of process disturbances by adjusting the deposition time. To assess the control approach, an artificial process disturbance was introduced as a $5^{\circ} \mathrm{C}$ temperature reduction for each successive wafer, resulting in a declining film weight. From the controller's perspective this was an unmodeled drift, which the control system would need to compensate. As input to the controller we could use either the $\mathrm{W}$ film weight or the HF signal from the preceding run, and the controller's task was to calculate a suitable deposition time for the following run so as to bring and maintain the film weight to its target value. The use of the HF RGA signal rather than the microbalance measurement as the control variable was of primary importance since the RGA was the sensor directly monitoring the process. Initial: 

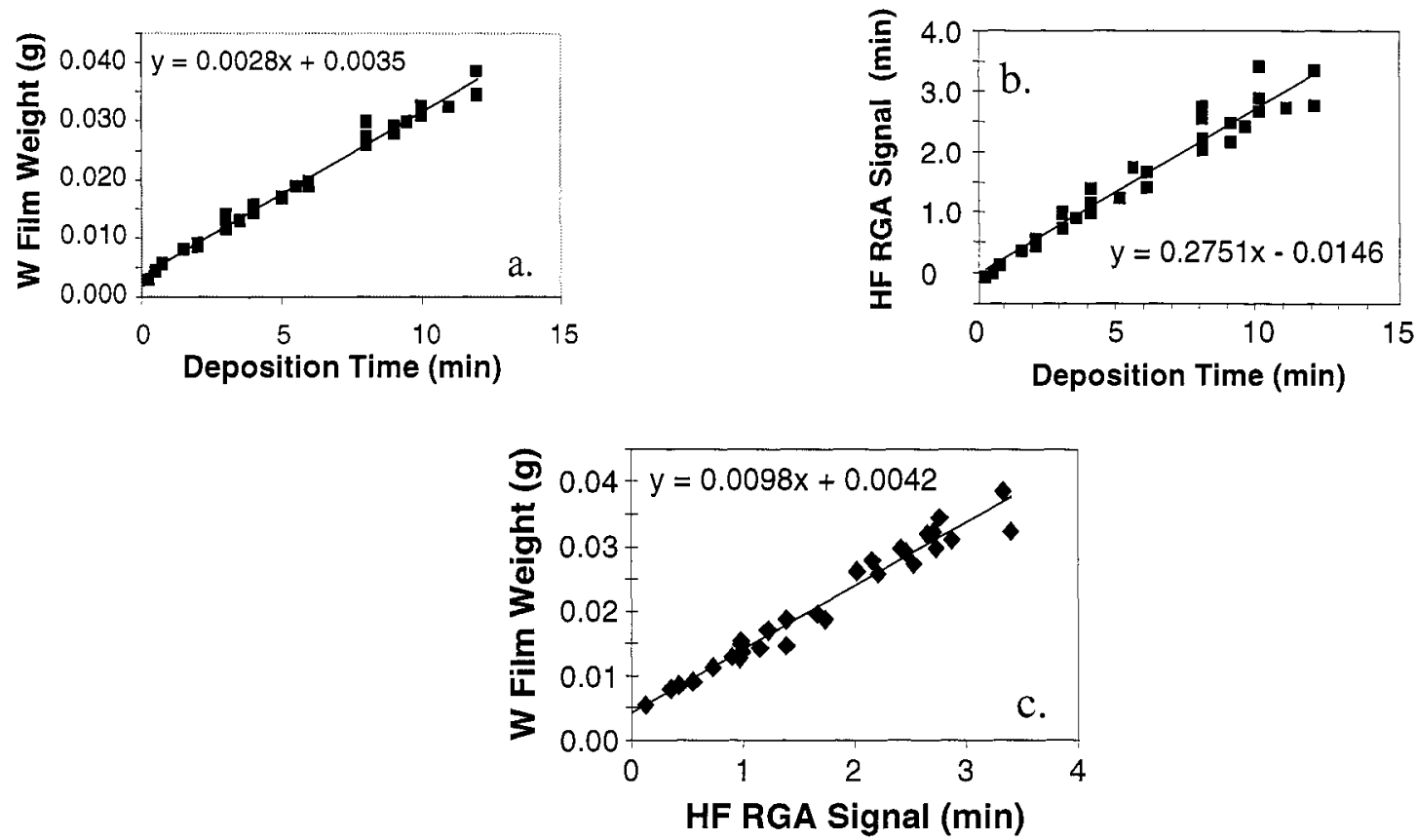

FIGURE 1. Linear static models for the process: (a) W film weight vs. deposition time; (b) HF product signal vs. deposition time; and (c) HF product signal vs. film weight. Model (c) is determined from models (a) and (b). The HF product signal included integration through the process cycle, background correction, and normalization [3].

experiments, however were performed with the microbalance so as to test the robustness of the control algorithm.

\section{DEVELOPMENT OF THE CONTROLLER}

The development of a successful control strategy required (1) a reasonably accurate process model and (2) the development and tuning of a suitable control algorithm

\section{Process Modeling}

We used three empirical models. (1) A model between the $\mathrm{W}$ film weight and the deposition time (Fig. 1a) was used with post-process weight measurements to test the control algorithm. (2) A model between the RGA HF signal and the deposition time (Fig. 1b) provided a control strategy in which the secondary control variable (in-situ sensor signal for $\mathrm{HF}$ product) could drive the manipulated variable (deposition time). (3) A model between the $\mathrm{W}$ film weight and the RGA HF signal (Fig. 1c) was derived from (1) and (2).

All three models were linear static models and were obtained by processing 37 wafers under identical conditions except for varying deposition times. The weight of the $\mathrm{W}$ film was determined post-process with an electronic microbalance. The data for the first wafer of each run was omitted so as to avoid the well documented "first wafer effect"[3] in the development of the models.

\section{Controller development and tuning}

The development of a robust control scheme depends on the existence of a process model that correlated the manipulated variable (here, deposition time) to the control variables, either primary (film weight) or secondary (HF product signal). A linear static process model can be represented by the equation:

$$
\tilde{y}_{n}=\widetilde{\gamma}_{n-1}+\widetilde{\beta} x_{n}
$$

where $\tilde{\gamma}_{n-1}$ denotes the $\mathrm{y}$ intercept of the model, $\widetilde{\beta}$ 
the slope of the model, $\mathrm{x}$ the manipulated variable, $\mathrm{n}$ the run number, and $\tilde{y}_{n}$ denotes the model output .

The actual process typically follows a somewhat different behavior, which is characterized in a different model known as the plant. This model is expressed by the equation:

$$
y_{n}=\gamma_{n}+\beta x_{n}
$$

Where $\gamma_{n}$ is the y intercept, $\beta$ the slope of the plant, and $y_{n}$ is the plant output. For control schemes using the Internal Model Control (IMC) approach, $\gamma_{n}$ can be interpreted as the load or disturbance at the output. The model slope $\widetilde{\beta}$ was taken as fixed but in general different from $\beta$ due to model uncertainty or error, and $x_{n}$ was the input that was manipulated to drive $y_{n}$ to the desired target. Finally, $\tilde{\gamma}_{n-1}$ was estimated using the plant data from the past $\mathrm{n}-1$ runs. Thus the controller updated the y-intercept term of the model after every run.

The exact controller type required depended on the type of disturbances which were expected and/or most important to compensate for. When step-like disturbances (shifts) were present in the process, a controller using an Exponentially Weighted Moving Average (EWMA) algorithm was adequate. Such a controller (known as TYPE I) calculated the value of the manipulated variable (deposition time in our case) for the following run by exponentially weighing the previous $\mathrm{n}-1$ run data using recursive relationships with one tuning parameter $\alpha$. However in the presence of drifts in the input variables, it could produce an offset which degraded controller performance to an extent depending on the modeling error, the amount of drift and the tuning parameter $\alpha$. For such processes, Adivikolanu and Zafiriou [4] have previously succeeded in incorporating the EWMA properties into an IMC structure, thus creating a more advanced and robust type of controller (known as Type II). Such a controller exhibited good model updating properties and zero steady state offset in the presence of process drifts, and the input variable value was calculated from recursive relationships that included two tuning parameters $\alpha$ and $\eta$.

For each controller, extensive simulations using Matlab were performed prior to each daily run to calculate the best values for the parameters, defined as the values that guaranteed smooth operation of the controller even in the presence of the worst case of

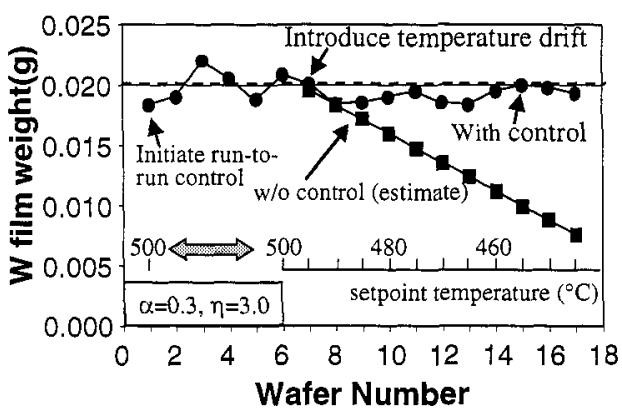

FIGURE 2. W film weight vs. run number. A target film weight of $0.02 \mathrm{~g}$ was set. After 6 wafers were processed, a temperature drift of $-5^{\circ} \mathrm{C}$ per run was experimentally introduced. The Type II run-to-run controller maintains film weight close to the target value, using the primary control variable (post-process film weight) to adjust the manipulated variable (deposition time).

mismatch between the process model and the plant model.

\section{RESULTS}

\section{Validation of the control strategy with ex- situ, post-process metrology}

A first objective was to test the behavior of the controller. For that purpose, we decided to use the $\mathrm{W}$ film weight as measured by the microbalance as the control variable. A target $\mathrm{W}$ film weight of $0.02 \mathrm{~g}$ was chosen, corresponding at a deposition time of $5.82 \mathrm{~min}$ (from the model relating weight of $\mathrm{W}$ and deposition time). During the first run the $\mathrm{W}$ film weight obtained was slightly different due to plant-model mismatch (see Figure 2). As a result the controller had to take action to bring the weight on the target value. This initial discrepancy was interpreted as a step like disturbance and a TYPE I controller was used to handle it. At the $7^{\text {th }}$ wafer, a temperature drift of $-5^{\circ} \mathrm{C}$ per run was introduced. In the absence of control, this drift would have resulted in declining film weight as "w/o control (estimate)" in Fig. 2; this estimate was based on the $\mathrm{W}$ film weight obtained from $5 \mathrm{~min}$ deposition experiments at the specific temperatures, extrapolated to the current deposition time. Once the temperature drift was introduced, the Type II controller - specifically designed to handle such unmodeled drifts - was used to calculate the required deposition times so as to maintain the specified film weight value. Figure 2 indicates that this was successful to about $3 \%$, which we estimated as the microbalance accuracy. 

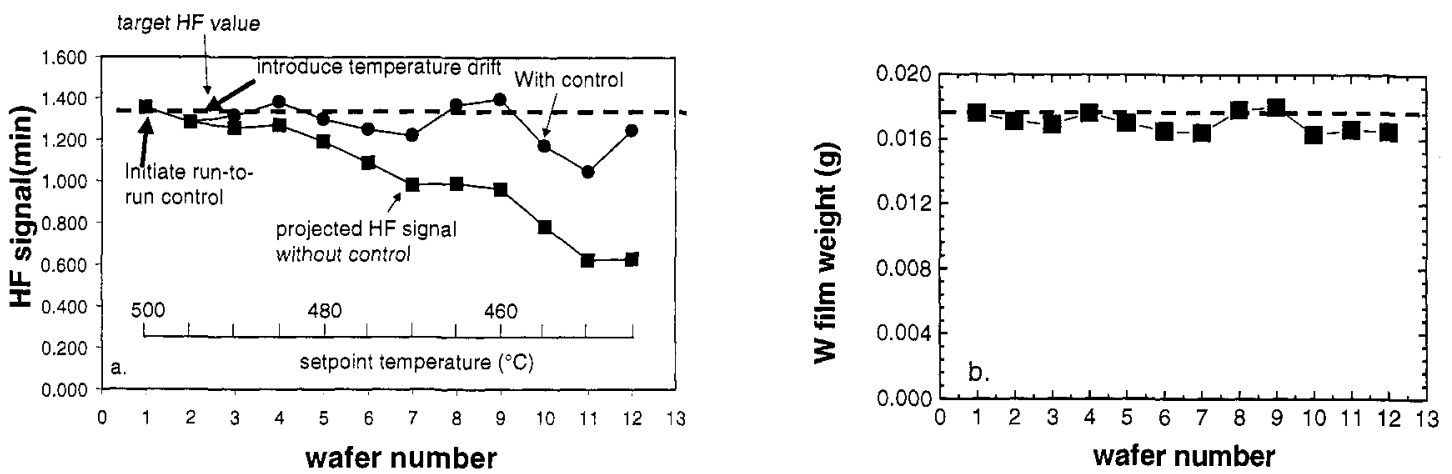

FIGURE 3. HF RGA signal vs. Run number. The initial run defines the target HF value and the temperature drift is introduced from the following run. The controller succeeds in keeping the HF normalized HF signal fairly close to the target while the resulting $\mathrm{W}$ film thickness ia also fairly stable.

\section{Applying run-to-run control with in-situ, sensor-based process metrology}

The real test for the potential use of sensor-based metrology as a basis for run to run control applications involves using in-situ mass spectrometry (RGA) signals as secondary control variables. For these experiments, we used the HF signal for the first wafer as the target value for the secondary control variable, and we initiated the temperature drift from the second wafer. Post-process measurements of film weight were carried out but not used for control. As can be seen in Figure 3a, the controller succeeded in keeping the HF normalized RGA signal within about $10 \%$ of the target value, as seen in the curve "with control", despite random process variations which caused temporary overshoot of the target value. The HF signal levels for deposition times varied for each wafer by the controller provided a means to estimate what the drift in HF signal would have been in the absence of control (by rescaling the signal inversely proportional to the manipulated deposition time); thus, the curve "projected HF signal without control" shows what the temperature drift would have caused in the absence of control.

The ultimate purpose of in-situ sensing and corresponding metrology using the secondary control variable is to achieve control of the primary variable, film weight, with sensing rapid enough to allow runto-run control. As seen in Fig. $3 \mathrm{~b}$, the $\mathrm{W}$ film weights resulting from run-to-run control of the in-situ HF metrology signal were fairly stable and consistent with the capabilities of the sensor-based metrology (about 7\% error) using mass spectrometry to sense HF product signals. There was also a small offset between actual weight averaged over the wafer batch and the target weight as defined by the first wafer.

\section{CONCLUSIONS}

These experiments demonstrate that wafer-state metrology based on in-situ mass spectrometry can produce effective run-to-run control in the presence of significant systematic process drift. Because the degree of control is consistent with the metrology capability, improvements in metrology accuracy are desirable and worthwhile. In the W CVD case studied here, the metrology is limited largely by the low conversion rate of reagents, so improved metrology and control are both anticipated for processes more typical of common manufacturing processes, where conversion rates are at least $10 \mathrm{X}$ larger. Further work is under way to assess the prognosis for in-situ sensorbased control in these domains.

\section{REFERENCES}

1 S. W. Butler, J. Vac. Sci. Technol. B 13(4),1917, (1995)

2 N. A. Chaudry, R. Telfeyan, B. Moore, H. Etemad, J. Moyne, SRC TECHON '93, 31,Sep 1993, Atlanta, GA

3 T. Gougousi, Y. Xu, J. N. Kidder Jr., G. W. Rubloff, and C. R. Tilford, J. Vac. Sci. Technol. B, 18(3) 1352 (2000).

4 S. Adivikolanu and E. Zafiriou, IEEE Trans. Electronics Packaging Manufacturing, 23(1), 56, (2000\}. 